Investigaciones Fenomenológicas, n. 9, 2012, 195-220.

e-ISSN: $1885-1088$

\title{
UN ANÁLISIS DE LA EVIDENCIA \\ EN LA LógICA Formal Y TRASCENDENTAL DE HUSSERL
}

\author{
Ivana Anton Mlinar \\ Universidad Nacional de Cuyo, Mendoza, Argentina \\ mlinariv@yahoo.es
}

\begin{abstract}
Resumen: Los aportes de Lógica formal $y$ trascendental en torno a las diferencias que se manifiestan en la evidencia de un juicio constituyen un paso fundamental en la descripción de la experiencia y de la evidencia en general, otorgando al camino unilateral de la expresión y la significación llevado a cabo hasta este punto, su lugar fenomenológico y su propia evidencia, a la vez que descubriendo e ingresando -a través del análisis genético- en la experiencia antepredicativa y su configuración tipificante -que será desarrollada en Experiencia y juicioen cuanto génesis y forma originaria de toda evidencia.
\end{abstract}

Palabras clave: Evidencia, lógica, juicio, Husserl.

\begin{abstract}
The contributions of Formale und transzendentale Logik around the differences that arise in the evidence of a judgement constitute a fundamental step in the description of experience and evidence in general, giving to the unilateral path of expression and meaning carried out till this point its phenomenological place and its own evidence, whereas at the same time discovering and entering -through the genetic analysis- the prepredicative experience and its typifying configuration -that will be developed in Erfahrung und Urteil- as genesis and originary form of all evidence.
\end{abstract}

Keywords: Evidence, logic, judgement, Husserl.

Ya resultaba claro a partir de estudios previos a la redacción de Formale und transzendentale Logik que las entidades lógicas tienen existencia ideal y que, además, nos son dadas con evidencia; de hecho, podemos experimentar la misma entidad ideal en una multiplicidad de actos diferentes ${ }^{1}$. Ahora bien, el problema radica en la fundamentación de su origen. Los análisis alcanzados en Filosofía de la aritmética y en Investigaciones lógicas no llegaban a dar cuenta precisamente del origen de estas entidades y elementos lógicos, aporte necesario de una teoría si pretende constituirse en teoría universal de fundamentación última de la ciencia. La Lógica procede didácticamente aunque no por ello es fácilmente comprensible en su exposición ${ }^{2}$. De allí que la Primera Sección

\footnotetext{
${ }^{1}$ Cfr. Hua XVII, 162. Las citas en español de Formale und transzendentale Logik corresponden a Lógica formal y lógica trascendental, México, U.N.A.M., 1962. Traducción de Luis Villoro.

${ }^{2}$ Lohmar sostiene que la Lógica posee una forma didáctica no habitual, por comenzar su exposición sin haber realizado la reducción trascendental -como sí es el caso en Meditaciones Cartesianas-, de tal manera que introduce paulatinamente al científico de la naturaleza, al matemático y al lógico en la fe-
} 
transcurra de modo deliberado sin la efectuación de la reducción trascendental, a fin de hacer manifiestos los objetivos y metas de la lógica que, incluso desde una actitud natural, señala hacia una dilucidación de las operaciones subjetivas constituyentes en las ciencias formales a partir de una triple estratificación en la constitución del sentido [§§ 12-22]. En este contexto hacen su aparición las diferencias en la evidencia que fundamentan los distintos niveles de la apofántica, es decir, los distintos modos de formular el juicio.

Con estos primeros análisis Husserl pretende advertir que cuando tenemos que ver con entidades lógicas no somos pasivos, sino que es necesaria una actividad originalmente productora [erzeugende Aktivität] a fin de que lleguen a darse estas formaciones lógicas mismas ${ }^{3}$, lo cual significa que todo juicio emitido es fruto de una génesis, posee una especie de historicidad en sí mismo que es el resultado de diversos sentidos y horizontes asumidos en la formación activa de los nuevos juicios.

Recorreremos, en primer lugar, los análisis en torno a la identidad del juicio, los modos de formulación y sus correspondientes diferencias en la evidencia, como aparecen en la Primera Sección, para continuar luego con los aportes de la Segunda Sección, donde las consideraciones genéticas alcanzan no sólo a los juicios sino a todo tipo de objetos, incluso a las cosas reales.

1. PRIMERA SECCIÓN: LA IDENTIDAD DEL JUICIO, LOS MODOS DE FORMULACIÓN Y LAS CORRESPONDIENTES DIFERENCIAS EN LA EVIDENCIA (CONFUSIÓN, DISTINCIÓN Y CLARIDAD)

"El mismo juicio puede estar dado con evidencia en muy diferentes modos subjetivos de darse ${ }^{\prime 4}$. Esta sentencia, que aparece muy frecuentemente en los textos de Husserl, contiene en gran medida el enigma de la relación entre evidencia, identidad [Identität] y mismidad [Selbigkeit].

En contraste con las Investigaciones lógicas, donde se trata en parte de afirmar la desprendida identidad de los significados con respecto a todos los actos del significar, en la Lógica se trata de fundamentar, por medio de una evidencia correspondiente, la identidad del juicio ligada a una manera de darse

nomenología trascendental a partir de sus propias experiencias en la actitud natural. Cfr. Dieter Lohmar, Edmund Husserls 'Formale und transzendentale Logik', Darmstadt, WBG, 2000, p. 1.

${ }^{3} \mathrm{Cfr}$. el título del $\S$ 63: La actividad originalmente productora como acto de darse las formaciones lógicas mismas. El sentido de la expresión "producción".

${ }^{4}$ Hua XVII, 61. 
- la identidad que atraviesa múltiples maneras de darse. Sin embargo, lo que no se expresa claramente $y$, por ello, resulta problemático, es en qué consiste esta "nueva" identidad del significado o, dicho de otra manera, qué es "lo mismo", que es dado con evidencia, si no se trata ya de la identidad de la especie (como en Investigaciones lógicas), y, correlativamente, qué es lo diverso que, paradójicamente debe ser llamado "mismo" [Selbst] y que constituye la correspondiente evidencia.

Siendo esta la problemática, ocupémonos de las tres clases de evidencia (confusión [Verworrenheit], distinción [Deutlichkeit] y claridad [Klarheit]) que se corresponden con tres conceptos de juicio ${ }^{5}$.

Cuando se presenta una ocurrencia vaga o un vago significado de una oración enunciativa leída, comprendida y asumida, no tiene lugar una emisión explícita de la espontaneidad judicativa. Esta mención confusa [verworren] se distingue [verdeutlicht sich] únicamente cuando se introduce un proceso de identificación impletiva, una actividad sintética producida desde el yo. Un juicio vago o confuso resulta entonces noemáticamente a partir de una unidad de significado de formaciones indicadas que, noéticamente, se corresponden con un complejo asociativo. En cuanto formación lingüística, el juicio vago es ya un presentarse originario como él mismo, aunque no un considerar temático del juicio, lo cual ocurre sólo en el proceso de distinción.

Husserl introduce aquí ${ }^{6}$ una observación importante: por un lado, el juicio mismo en cuanto juicio puede llegar a la dación de sí mismo [zur Selbstgegebenheit kommen], es decir, convertirse en un juicio distinto; por otro lado, el estado de cosas, en cuanto aquello hacia lo cual quiere dirigirse el que juzga "por medio de" su juicio, puede llegar a la dación de sí mismo. De esta manera, hay aquí dos evidencias: la primera es la evidencia de la distinción; la segunda, la de la claridad. "[L]a evidencia de la mismidad [Selbigkeit] de un juicio puede pasar por modos de darse esencialmente diferentes" ${ }^{\prime 7}$.

Por su parte, un juicio claro puede ser emitido en la claridad de la anticipación o en la claridad de la posesión. La claridad de la anticipación no da el estado de cosas mismo, sino que lo mienta pre-figurado [vor-verbildlichend]. Esta

\footnotetext{
${ }^{5}$ Este análisis se despliega temáticamente en §§ 12-22.

${ }^{6}$ Cfr. Hua XVII, 65.

7 Ibidem, pp. 65s. Corregimos la traducción de Selbigkeit (asumimos mismidad en lugar de identidad, como aparece en la versión española) para evitar la confusión con el término Identität.
} 
anticipación intuitiva puede ser confirmada en la posesión, lo que se llama precisamente un juicio evidente $[\S 16 \mathrm{c})]^{8}$.

En lo que sigue indagaremos más precisamente qué es lo dado, en realidad, en cada modo y en qué consiste el respectivo "sí mismo" [Selbst] de cada evidencia. Por otro lado, para afirmar algo como "el mismo juicio en diversas maneras de darse", es fundamental identificar "el mismo juicio".

Tomemos en primer lugar la confusión [Verworrenheit]. Husserl considera bajo este modo diversas clases de maneras de darse, como, por ejemplo, lo leído descuidadamente, la retención, la rememoración y la ocurrencia aperceptiva [Apéndice II,$\S 3 \mathrm{c}$ ) $]^{9}$. Si modos tan diversos como éstos son denominados confusos, debemos encontrar lo esencial en ellos, que hace de cada uno una mención confusa. Debemos antes resaltar una diferencia importante dentro de este modo, a saber:

1. una confusión del comprender al revivir [Nachverstehen], cuando se trata de comprender a los otros, es decir, leer o escuchar -se muestra, por principio, aperceptivamente-, y

2. una confusión del juzgar propio originario y sus diversos modos, es decir, la confusión del enlazar con anteriores adquisiciones de juicio, como en el caso de la retención y de la rememoración.

En cuanto a 1: En la lectura o escucha desatenta nos es dada una unidad sensible, una copertenencia del signo, pero no es producido sintéticamente un juicio distinto. ¿En qué consiste el presentarse de un Selbst para asumir una evidencia? Lo dado aquí en sí mismo es precisamente esta unidad sensible con su contenido de sentido, la formación gramatical que, de ninguna manera, es confusa ni puede serlo, si es que debe ser posible una teoría de las formas [Formenlehre] fundada en este nivel. Lo confuso es, por el contrario, el juicio que, eventualmente, debe distinguirse o que aún no ha sido distinguido, y que es indicado por medio de signos lingüísticos y es dado aperceptivamente. La pregunta surge por sí misma: ¿debemos hablar efectivamente de una evidencia de la confusión? ¿Debemos considerar lo dado confusamente, según la concepción del mismo Husserl, como evidencia, si la evidencia, por principio, debe valer como norma metódica de la cientificidad?

\footnotetext{
${ }^{8}$ Cfr. Ibidem, pp. 66s.
}

${ }^{9}$ Cfr. Ibidem, p. 321. 
Heffernan plantea la cuestión preguntándose -en el marco del análisis del $\S$ 16- si la ocurrencia aperceptiva, en cuanto evidencia confusa, es una evidencia, "sólo" una evidencia secundaria o ninguna evidencia en absoluto. Concluye que es una evidencia y, a la vez, no lo es. En relación con la confusión del juicio en cuanto tal, si se considera que dentro de la confusión misma se dan grados, mientras más se acerque la ocurrencia aperceptiva al polo de la "pura" confusión, tanto más no será evidencia; y, mientras más se acerque al polo de la distinción "pura", tanto más será una evidencia. De modo que la confusión de la ocurrencia aperceptiva puede pero no necesariamente debe ser una evidencia. Por otro lado, si por evidencia se entiende el darse las cosas mismas en contraposición al darse los "meros" juicios, en este sentido tanto la confusión como la distinción no podrían valer como "evidencias"10.

Antes de ofrecer una respuesta a la pregunta ya formulada acerca de si la confusión puede o no considerarse como una evidencia, consideremos las otras formas de confusión.

Con respecto a 2: Si la ciencia es poseída como un acervo de adquisiciones permanentes de conocimiento, Husserl debe encontrar el fundamento para la justificación de su confianza [Apéndice II, § 7] $]^{11}$. La retención y la rememoración son formas propias de conciencia que permiten la apropiación de la ciencia. En esto radica el interés de identificar la evidencia contenida en ellas.

La confusión en estos dos modos consiste en el carácter no-original de sus maneras de darse algo. Y, si bien ello vale como rasgo fundamental de la confusión en general, puede diferenciarse el caso 2 del caso 1 en la ausencia de algo dado aperceptivamente. Se trata de la conservada adquisición de la constitución de una objetividad de conciencia originalmente efectuada, con la que se enlaza pasivamente.

E incluso desde la perspectiva de la materia podrían distinguirse algunos puntos. En el proceso de distinción de lo dado aperceptivamente (caso 1), la materia experimenta una especie de "aumento", si se nos permite la expresión. El proceso de distinción trae consigo una nueva objetividad: el juicio emitido, pues lo gramatical anteriormente dado con evidencia se convierte en una parte

\footnotetext{
${ }^{10} \mathrm{Cfr}$. George Heffernan, Isagoge in die phänomenologische Apophantik. Eine Einführung in die phänomenologische Urteilslogik durch die Auslegung des Textes der Formalen und transzendentalen Logik von Edmund Husserl, Dordrecht / Boston / London, Kluwer, 1989, pp. 88ss.

${ }^{11}$ Cfr. Hua XVII, 325s.
} 
dependiente del juicio surgido. Pero no puede hablarse del mismo juicio, pues no había ningún juicio -en cuanto sentido sin contradicción- en absoluto antes de haberlo emitido (distinción). Sin embargo, no ocurre de la misma manera en los otros modos: puesto que la retención y la rememoración enlazan con un juicio ya dado en sí mismo una vez, la materia se conserva y tiene lugar más bien un cambio cualitativo.

Precisamente en esta diferencia fundamental creemos que radica el motivo por el cual Husserl puede considerar estos dos modos de la confusión como, no obstante, evidentes, y que permita que funcionen como condiciones de posibilidad de la ciencia. La evidencia legitimada consiste aquí -en el caso 2- en un "Selbst", por así decir, conservado, es decir, lo conservado resulta legitimado, pues se trata de un Selbst ya dado. En consecuencia, nos parece que, por el contrario, no se presenta como evidencia lo que podemos denominar "confusión misma", es decir, lo dado aperceptivamente del comprender reviviendo [Nachverstehen] (caso 1), pues lo propiamente confuso no es la formación gramatical, que, como objetividad dada (es decir, como forma sintáctica) encuentra su cumplimiento (podríamos decir que es dada de manera clara), sino el juicio no emitido, la posicionalidad de su contenido (considerado desde la distinción), que, en este sentido, sí presenta idéntica materia que el juicio distinto, como se comprenderá más abajo.

Si bien Husserl habla de modos de emisión [Vollzugsmodi] para distinguir confusión, distinción y claridad, nos atrevemos a expresarlo también de la siguiente manera: lo confuso mismo del caso 1 reside en el "qué" (es decir, el sentido que no es dado distintamente, pues se atiende solo a la forma sintáctica), mientras que lo confuso de la retención y de la rememoración se encuentra en el "cómo" (es decir, en el carácter del acto, con el que se corresponde la ausencia de la cualidad ponente, que se asumirá en el proceso de distinción).

Un juicio confuso es un juicio que, en cuanto juicio, es solo mentado, es un juicio no-explícito ${ }^{12}$. Precisamente en el proceso de distinción [Verdeutlichung] es cuando el juicio mismo es dado, cuando la mención misma es intuida: "el juzgar 'distinto', explícito, es la evidencia propia del 'juicio distinto', en cuanto éste es la objetividad ideal que se constituye originalmente en esa acción sinté-

${ }^{12}$ Cfr. Ibidem, p. 63. 
tica y es identificado al repetirse ${ }^{13}$. En este proceso pueden darse dos opciones: o bien es dado lo no contradictorio, o bien lo contradictorio. En el primer caso encuentra cumplimiento la unidad de sentido meramente mentada en la confusión, es decir, los miembros implicados en la unidad total del juicio se muestran compatibles ${ }^{14}$, no se contradicen entre sí, de manera que cada intención parcial de la creencia anteriormente no explícita encuentra explicitación en el proceso de distinción. En el segundo caso, el de la contradicción, se presupone la unidad total de la creencia a partir de una mención total, pero, a partir de la supresión de una determinada creencia particular, se ve suprimida la creencia total. Hay una identidad de materia a pesar del cambio que tiene lugar por la cancelación de la creencia: como en el caso en que no comparto la creencia de otro y, sin embargo, tengo la "mera representación"15, comprendo el sentido de tal contenido.

Ahora bien, en vistas al estado de cosas mentado en el juicio, tanto el juicio confuso como el distinto son conciencias vacías.

Lo expuesto permite aclarar algo que Husserl no siempre distingue, aunque se advierte como su intención. Si la lógica formal pretende ser la teoría universal y fundante de la ciencia, debe poder justificar todos sus pasos. Por medio de la reflexión acerca de las operaciones subjetivas que están a la base de las formaciones objetivas de la lógica (que se explicita en tres disciplinas: la teoría de las formas, la lógica de la consecuencia y la lógica de la verdad), deberá mostrarse la evidencia presente en ellas para alcanzar esa justificación. Cada disciplina cuenta con su propio objeto que, claro está, va engarzándose, de manera sintética a modo de parte dependiente, con la objetividad -o sentido enriquecido- de la disciplina del grado superior. De esta forma, cada estrato de operaciones subjetivas se diferencia por una evidencia correspondiente. $Y$ esto quiere decir que cada estrato posee su propia intención vacía y un determinado estilo de cumplimiento de esta intención que caracteriza esa evidencia; en otras palabras, "las respectivas intenciones de un juicio confuso y de un juicio distinto (y claro anticipativo) pueden ser cumplidas (i. e. evidentes) o decepcionadas sin considerar la evidencia del estado de cosas mismo"16.

\footnotetext{
13 Ibidem, p. 65.

${ }^{14}$ Cfr. Ibidem, p. 59.

${ }^{15}$ Cfr. Ibidem, p. 64.

${ }^{16}$ Dieter Lohmar, op. cit., p. 50.
} 
En su análisis de estos parágrafos, Heffernan comenta que se trata precisamente de dos actos diversos de juzgar a los que corresponden dos juicios: en el juzgar en confusión se constituye el juicio confuso; en el juzgar en distinción se constituye el juicio distinto. Se trata de dos conceptos de juicio que poseen un significado diferente ${ }^{17}$. Por medio del fundamento sensible que ofrece la escritura y el lenguaje son dadas las expresiones. Estos elementos se configuran pasivamente como indicaciones que se unifican en vistas a la mención de un juicio. En la evidencia de la confusión la mismidad dada es precisamente esa unidad gramatical, fundamento de la expresión y de la unidad de mención de un juicio. Vale el recuerdo de Heffernan ${ }^{18}$ acerca de la diferenciación -que ya exponía Husserl en los Prolegómenos y en Investigaciones lógicas- entre proposición [Satz] -como el ámbito de los signos que "indican" - y juicio [Urteil] o significado [Bedeutung] -el ámbito de los significados que "indican"-: la vaguedad o confusión y la distinción pueden tener lugar tanto en la expresión como en el juicio, lo que daría las siguientes posibilidades: 1) la expresión es confusa y el juicio es confuso, 2) la expresión es confusa pero el juicio es distinto, 3) la expresión es distinta pero el juicio es confuso y 4) la expresión es distinta y el juicio es distinto.

Pero, ¿qué es finalmente lo dado en la evidencia de la confusión y qué en la evidencia de la distinción y cuál es "el mismo juicio" que "puede estar dado con evidencia en muy diferentes modos subjetivos de darse" $[\S 16 \mathrm{a})]^{19} \mathrm{si}$, en cada caso, se trata de dos juicios, según se expuso? Solo desde el punto de vista de un análisis genético -que es introducido explícitamente en la Segunda Secciónadquiere sentido y unidad esta consideración, en la que se vuelven fundamentales las transiciones o pasos de un modo a otro. Si bien Heffernan advierte en su comentario la importancia de las transiciones -pues la estructura fundamental en la que adquieren su sentido los niveles y estratificaciones de la lógica y de las operaciones subjetivas son las maneras de intención y cumplimiento respectivos ${ }^{20}$, no da con la consecuencia de tal consideración: la evidencia de la confusión, la evidencia de la distinción y la evidencia de la claridad son tales genéticamente consideradas desde la dación del estado de cosas, pues lo dado

\footnotetext{
17 George Heffernan, op. cit., p. 81.

${ }^{18}$ Ibidem, pp. 83ss.

${ }^{19}$ Ibidem, p. 61.

${ }^{20}$ Cfr. Ibidem, pp. 77-79.
} 
en cada nivel (objeto propio de las diversas disciplinas lógicas), en cuanto tal, es dado o, más bien, puede ser dado, con "claridad" 21 , es decir, ser intuido según el estilo propio exigido por la objetividad correspondiente. Tampoco Husserl es explícito en este sentido, aunque necesariamente se desprende esta conclusión (que es más bien el presupuesto de los análisis de estos parágrafos de la Lógica) de las siguientes tesis que sí son asumidas en la exposición:

1. Una teoría de las formas o gramática puramente formal tiene por objeto "un sistema concluso de formas fundamentales", "el sistema de todas las formas concebibles de juicio"22, lo cual implica que, en cuanto objetividad, una gramática pura debe poder ser mentada y dada en intuición, es decir, encontrar su cumplimiento propio.

2. La lógica de la consecuencia es un "nivel superior de la lógica formal del juicio"23. Cuenta, a su vez, con su propio objeto: ella es "la ciencia de las formas posibles de juicios verdaderos" 24 , se trata de "las leyes lógicas que regulan la mera no contradicción de los juicios basadas en su forma." 25

3. Otro tanto con la lógica de la verdad ${ }^{26}$.

4. Por otro lado, puede tener lugar la identidad del juicio a través de diversas maneras de darse -como ya se ha mencionado.

Heffernan entiende que la identidad y la diferenciación de los juicios constituyen las dos caras de una misma moneda, ya que, resaltando las transiciones de uno a otro modo de formulación del juzgar o del juicio es dada la única posibilidad de mostrar no sólo el hecho de que sino cómo la identidad o la diferenciación de la formación del juicio puede atravesar actos esencialmente idénticos o diversos del juzgar. Sólo una teoría de la evidencia puede explicar que los diversos modos de formulación del juicio, ellos mismos, son los que pueden "mezclarse" o "entrelazarse"27. Con todo, esta argumentación no explica la evidencia propia de cada disciplina, pues, si bien es cierto que las diferentes evi-

\footnotetext{
${ }^{21}$ Las comillas quieren señalar la diferencia entre la "claridad" como la dación propia que puede alcanzar un juicio en el estrato de la morfología (o de la lógica de la consecuencia) y la claridad propiamente dicha en el proceso de aclaración del juicio.

${ }^{22}$ Ibidem, p. 55.

${ }^{23}$ Ibidem, p. 58.

${ }^{24}$ Idem.

${ }^{25}$ Ibidem, p. 59.

${ }^{26}$ Nos detendremos más detalladamente en esta disciplina y en sus implicancias para la evidencia en lo que sigue.

${ }^{27}$ Cfr. Ibidem, pp. $76 \mathrm{~s}$.
} 
dencias fundamentan los distintos niveles de la apofántica ${ }^{28}$, no puede decirse, por ejemplo, que la morfología da su objeto sólo con evidencia de la confusión o distinción ${ }^{29}$, sino sólo cuando su objeto se vuelve momento, esto es, mención, de otra objetividad: el sentido unitario de un juicio. Aquí se encuentra en juego aquel método de la aclaración introducido en Ideas $I^{30}$ : la objetividad es ahora el juicio mismo, de tal manera que, en cuanto noema, cuenta con sus diferentes estratos de constitución que, correlativamente se corresponden con diversos modos de formulación del juicio y con la multiplicidad de actos de juicio en los que se constituye ${ }^{31}$. Cada estrato puede ser, a su vez, mentado y cumplido según su propio estilo correspondiente a la objetividad dada (formas, significados o cosas).

El problema de la exposición de esta sección radica en que se manejan diversos significados de juicio que dificultan la diferenciación e identidad que buscamos, como ya señalamos antes a partir de las diversas posibilidades de combinación de confusión y distinción con respecto al juicio y a la expresión. Como lo que está en juego aquí es la fundamentación de la lógica formal como apofántica formal, el concepto predominante de juicio, esto es, el juicio que debe fundamentarse, es un juicio lingüístico, es el juicio distinto, el juicio formulado por medio de una operación categorial, de tal manera que la expresión se convierte en la articulación o esquema que rige la configuración y constitución de los juicios, ya sea siguiendo el esquema de las palabras en el caso de la confusión, o, siguiendo y efectuando este mismo esquema en el caso de la evidencia de la distinción: "Si los juicios vagos no estuvieran articulados por la articulación sensible de los signos verbales, no sería posible, en modo alguno, morfología ni lógica"132.

Ahora bien, por otro lado, Husserl sostiene más adelante [§ 21] que "el mismo juicio" del que se trata, es decir, "el concepto más amplio de juicio", es "el que no está afectado por las diferencias entre confusión, distinción y clari-

${ }^{28}$ Cfr. el título mismo del $\S 16$ [Lógica formal y lógica trascendental, p. 61]: Las diferentes evidencias que fundamentan distintos niveles de la apofántica. Evidencia clara y evidencia distinta.

${ }^{29}$ Cabe mencionar que en el $\S 70$ se atribuye la evidencia de la distinción a la morfología como primera disciplina lógica: "... en la morfología basta simplemente con la distinción de la secuencia rítmica de las indicaciones verbales, para que los juicios estén dados con evidencia."

${ }^{30} \mathrm{Cfr}$. Edmund Husserl, Ideas I, §§ 67 y 68.

${ }^{31} \mathrm{Cfr}$. $\$ 57$ donde también vuelve a hacerse referencia a la unidad del juicio.

32 Hua XVII, 75s. Cfr. Dieter Lohmar, op. cit., pp. 54-56, donde se señala y argumenta que la confusión y distinción tienen como punto en común de orientación la organización sensible y la configuración propias de la expresión lingüística. 
dad", y, puesto que todo juicio claro es también distinto y puede volverse confuso y, a la vez, a cada juicio distinto corresponde un juicio confuso como el mismo, "el concepto de juicio confuso abarca en cierto modo todos los juicios en su sentido más amplio, incluso los que pueden volverse distintos y claros ${ }^{\prime \prime 3}$.

Con todo lo expuesto vemos surgir una "tercera evidencia" y "un tercer concepto de juicio" ${ }^{34}$ que podríamos caracterizar como transversal ${ }^{35}$ y que -creemos- serán los que puedan finalmente dar cuenta de la diferenciación de los estratos propios correspondientes a cada disciplina lógica -con sus respectivas objetividades y formas propias de mención y cumplimiento- y de la identidad que, por otro lado, permite hablar de un mismo juicio que transita diversas maneras de darse.

No todo juicio confuso encuentra su correspondiente identificación con un juicio distinto. Sin embargo, cuando es el caso, el juicio confuso y el juicio distinto guardan una relación entre sí -sostiene Husserl- como la que corresponde a un juicio distinto vacío y a un juicio distinto pleno, es decir, el juicio distinto se constituye en "exposición evidente de la mención verdadera" ${ }^{36}$, dando paso, a su vez, a la "evidencia [d]el ser posible o verdadero de las cosas a que tiende el juzgar cognoscitivo" ${ }^{37}$, es decir, disponiéndose para la evidencia de la claridad. Esto significa que sólo el juicio confuso que presenta una tendencia a la distinción por su interés teórico y que, en consecuencia, se constituye en un juzgar cognoscitivo, es el que propiamente podrá denominarse confuso, distinto y claro en su proceso de dación que atraviesa diversos modos de formulación hasta llegar al cumplimiento o dación del estado de cosas al cual, por medio del juicio, se tiende. Pero el juicio propio del nivel de la confusión, digamos: un juicio propio de la morfología, $y$, otro tanto con un juicio propio de la lógica de la consecuencia (como también un juicio claro anticipativo), pueden ser cumplidos o decepcionados, es decir, pueden presentarse cada uno como intención (mención) o cumplimiento (juicio pleno) sin que haya referencia o se considere la dación o evidencia de un estado de cosas en la intuición. El plano de la evidencia de la confusión no depende, entonces, de su posibilidad o no de volverse

${ }^{33}$ Hua XVII, 73s.

${ }^{34}$ Ibidem, p. 73.

${ }^{35}$ Como bien señala Lohmar (cfr. op. cit., p. 60), no debe asociarse esta denominación de "tercera evidencia" o "tercer concepto de juicio" a la evidencia de la claridad de la anticipación. De allí nuestra caracterización de "transversal".

${ }^{36}$ Hua XVII, 73.

37 Ibidem, p. 74. 
evidencia de la distinción.

Es este "tercer concepto de juicio" o, primeramente, del juzgar, el que permite que se advierta la síntesis de coincidencia que hace de cada nivel una intención parcial de la intención total que alcanza su cumplimiento en un proceso de grados que va de una mayor lejanía (intuitiva) a una cercanía de lo dado. Correlativamente, es esa "tercera evidencia" la que tiene lugar en las síntesis de coincidencia en cuanto a la materia ${ }^{38}$. Es una coincidencia de identidad, no de cumplimiento, de tal manera que cada nivel, por ejemplo, una intención distinta [deutlich], asume en coincidencia de identidad la materia propia de la mención confusa, ampliada con su mención distinta propia: la consecuencia o no contradicción. Aquí se comprende por qué el juicio idéntico, "el mismo juicio" que recorre las diversas maneras subjetivas de darse, es el juicio confuso: la materia propia del juicio confuso es la mismidad que permite la identidad en las transiciones de una intención parcial a otra de grado superior. Aunque, a su vez, esa materia enriquecida del juicio distinto es la que pasa a identificarse con la materia del juicio claro de anticipación que se "engrosa" con un nuevo estilo de mención y de evidencia posible.

Toda esta exposición que tiene lugar en la Primera Sección recorre el camino o proceso de análisis de cualquier lógico, esto es, en actitud natural se parte desde la evidencia de las objetividades lógicas ya constituidas con las que se opera. Es por eso que, en el nivel de la morfología y de su evidencia de la confusión se recurre casi con exclusividad al caso de la ocurrencia aperceptiva [apperzeptiver Einfall $]^{39}$, pues la retención y la rememoración -consideradas más detenidamente y de modo referencial en el Apéndice $I I-$ manifiestan con mayor transparencia su carácter de "modificaciones intencionales"40, es decir, su ser secundario en referencia a una dación más originaria: la experiencia. Los análisis genéticos de la Segunda Sección traen a la luz el carácter fundamental de la

${ }^{38}$ Cfr. Ibidem, p. 64. Dice Lohmar que "las síntesis de identificación que conciernen a las síntesis de identidad, es decir, 'la coincidencia de identidad' concierne, sin embargo, sólo a la materia del juicio. De allí que ella permanezca independiente de su cumplimiento $y$, con esto, también independiente de la cualidad de acto edificada sobre ello" (op. cit., p. 50).

${ }^{39}$ La constitución de la ocurrencia aperceptiva (cfr. el comienzo de este capítulo, donde se dan ejemplos) hace más natural la visualización del proceso de aclaración pero no permite reconocer tan fácilmente su manera de darse pasiva y secundaria -en esta Primera Sección se trata, de hecho, de resaltar la evidencia propia de este nivel-, como sí es el caso en la retención y la rememoración, motivo por el cual se convierten estos últimos en referentes para el análisis genético. La ocurrencia aperceptiva se considera ahora un análogo de la ocurrencia rememorativa pasiva (cfr. Apéndice II, § 3 c, p. 321. Hua XVII, 321).

${ }^{40}$ Hua XVII, 314. 
retención y de la rememoración -más que de la ocurrencia aperceptiva- y de sus evidencias respectivas, pues son estas dos formas de conciencia las que posibilitan la ciencia en cuanto "contingente de adquisiciones permanentes de conocimiento, de evidencias que pueden en cualquier momento reactivarse ${ }^{\prime 41}$.

\section{La claridad}

El $\S 16$ b) comienza con una formulación que compendia en cierto sentido puntos fundamentales ya expuestos: "Mas también tomamos en cuenta una mezcla de otro tipo y, al depurarla, otra contraposición más importante: la mezcla y la diferencia, concebida con pureza, entre 'distinción' y 'claridad'"142. Mezcla y contraposición o diferencia. Otra mezcla y contraposición es la que tiene lugar entre la confusión y la distinción ${ }^{43}$. Cada uno de estos niveles de análisis -como ya se ha expuesto- se constituye, por un lado, con su propio estilo de mención y cumplimiento y, en consecuencia, con su correspondiente evidencia; y, por otro lado, cada uno de ellos puede constituirse como intención parcial de una mención total -el ya comentado "tercer concepto de juicio"- que encuentra en la denominada "tercera evidencia", como síntesis de coincidencia o identificación, su unidad y justificación. De allí que sostenga Husserl que "la evidencia de la identidad [Selbigkeit] de un juicio puede pasar por modos de darse esencialmente distintos" ${ }^{\prime 4}$. Es esta evidencia la que permite hablar de "un mismo juicio".

A partir del cumplimiento del "juzgar" confuso -que no es propiamente una efectuación activa-, es decir, al constituirse una unidad gramatical capaz de sentido, puede tener lugar el juzgar explícitamente, que, sin embargo, no es aún el juzgar con claridad. Este último tiende a la dación de las cosas mismas o situación objetiva. Ahora bien, la claridad del juzgar puede ser una claridad de la anticipación o una claridad de la posesión ( $\S 16$ c)). Cuando se juzga explícitamente -juzgar distinto-, el proceso de distinción puede dar lugar a "la clarificación del juicio en cuanto mención" ${ }^{\prime 45}, y$, en este sentido, Husserl ve en la claridad anticipativa una prefiguración [Vorverbildlichung] de la situación objetiva en cuanto anticipación intuitiva ${ }^{46}$. Lohmar señala que la figuración analógica

\footnotetext{
${ }^{41}$ Ibidem, p. 326.

42 Ibidem, p. 65.

${ }^{43}$ Cfr. Idem. "Confusión y distinción del juzgar pueden naturalmente mezclarse...".

${ }^{44}$ Idem. El resaltado es nuestro.

45 Ibidem, p. 66.

${ }^{46}$ Cfr. Idem.
} 
[analogische Verbildlichung] puede ser entendida como un caso especial de intuición, es decir, del darse ello mismo, pues la semejanza de la imagen [Bild] con la cosa permite que al menos algunas características de la cosa misma sean presentadas, a diferencia de la intención signitiva. También en los §§ 21 y 22 de la Sexta Investigación lógica trata Husserl, a su vez, la figuración analógica como un caso de actos intuitivos. Pero agrega Lohmar que Husserl no parece estar muy decidido en este sentido, pues más adelante (en el § 45) opone la presentificación imaginativa [bildliche Vergegenwärtigung] y el pensar puramente significativo o intención signitiva a la intuición ${ }^{47}$. Es necesario advertir que en el marco de su exposición de la claridad de la anticipación Husserl siempre recurre a los términos pre-figuración, pre-figurante, pre-figura [Vorverbildlichung, vor-vebildlichend, Vor-bild], etc., de modo que no sería del todo acorde exponer su interpretación de este juzgar como el que "intenta figurar [verbildlichen] el estado de cosas mentado", como sostiene Lohmar ${ }^{48}$. El recurso de Husserl a la figura o imagen (no casualmente utiliza "pre-figura") en este contexto $^{49}$ es más bien comparativo o analógico. La aparente indecisión acerca de la condición de acto intuitivo o no de la figuración viene a confirmar tanto la diferencia como la transición identificante que tiene lugar entre los estratos: así como la conciencia imaginativa da su propio objeto -la imagen- según una forma propia de mención y cumplimiento y, a su vez, en vistas a la cosa misma de la que es imagen se constituye como conciencia analógica que no da en persona, así también la claridad de la anticipación da la posibilidad de la verdad de los juicios, no aún la posesión intuitiva de la cosa. No puede decirse propiamente que sea una anticipación figurativa. Lohmar sostiene que aparentemente el autor habría tenido al comienzo del parágrafo un programa que luego no logra concretar: "la evidencia de la claridad anticipativa sería una anticipación prefigurativa, intuitiva de aquello hacia lo cual tiende el que juzga en el conocimiento. En esta evidencia se volvería intuitivo de modo pre-figurativo lo que la intención de conocimiento del juicio podría cumplir si llegara a intuición real" ${ }^{250}$. Insistimos en que este recurso de Husserl parece más bien una simple comparación -poco feliz, tal vez-, pues la claridad de la anticipación es una intención

${ }^{47}$ Cfr. Dieter Lohmar, op. cit., p. 56 y nota 82.

${ }^{48}$ Cfr. Ibidem, p. 56.

${ }^{49}$ Cada vez que aparecen estas palabras figuran, por cierto, resaltadas.

50 Dieter Lohmar, op. cit., p. 57. 
signitiva, que se cumplirá con la dación del estado de cosas mismo.

Los dos modos de la claridad (de la anticipación y de la posesión) presentan grados. Aquí quisiéramos llamar la atención sobre la nota al pie que ofrece el autor en el apartado c) en relación con este aspecto. La gradación -se dice- no se presenta con un límite, sino que se constituye como un sistema infinito de experiencias posibles por implicación intencional. $Y$ este sistema infinito como anticipación infinita tiene su propia evidencia ${ }^{51}$. Entendemos que ésta es la evidencia de la claridad de la anticipación. ¿Y por qué puede ser una evidencia esta anticipación vacía? La claridad perfecta es la claridad del ver, del aprehender la cosa misma; la claridad de la anticipación prefigura con perfección el objetivo al que tiende el juzgar, como ya se dijo. Heffernan advierte que la exposición de Husserl daría a entender que la evidencia de la claridad de la anticipación es "intuitiva" [anschauliche] pero que no "da en sí misma" [selbstgebend], lo cual implicaría que los conceptos "intuición" y "darse en sí mismo" o los conceptos "evidencia" y "darse en sí mismo" no coinciden. Debemos recordar aquí lo que se dice en el $\S 67$ de Ideas $I$, que gira precisamente en torno al "Método de la aclaración. 'Cercanía de lo dado' y 'lejanía de lo dado'":

\begin{abstract}
Una conciencia en que se da algo en el sentido pleno del término y una conciencia intuitiva, frente a una conciencia no intuitiva, o una conciencia clara, frente a una conciencia oscura, son una misma cosa. [...] Pero en todo esto no hay que entender el darse en el sentido de un darse originario, por consiguiente tampoco como un darse en la percepción. No identificamos lo "dado en sí mismo" [selbstgegeben] con lo "dado originariamente" [originär-gegeben] "en persona" [leibhaft]. En el preciso sentido señalado son una sola cosa "dado" [gegeben] y "dado en sí mismo" [selbstgegeben], estando el empleo de la expresión pleonástica destinado exclusivamente a servirnos para excluir el darse en su sentido más amplio, en el que se dice en definitiva de todo lo representable que se da en la representación (pero "de un modo vacío"). ${ }^{52}$
\end{abstract}

"Intuitivo", "dado" y "dado en sí mismo" son una y la misma cosa cuando se atiende a lo dado en cada nivel, según el estilo propio de cada objetividad (forma, significado o cosa). Ahora bien, la intención cognoscitiva, el conocimiento actual, reclama como meta el darse las cosas mismas. Es entonces que, desde la claridad perfecta ${ }^{53}$, la claridad de la posesión, se distingue una mayor o menor cercanía -o lejanía- de lo dado, es decir, se distinguirá lo dado origi-

${ }^{51}$ Cfr. Hua XVII, 66.

52 Hua III, 142.

${ }^{53}$ En varios lugares y, concretamente en la nota al pie ya mencionada, explica Husserl que la claridad perfecta se constituye en la experiencia externa como idea regulativa en sentido kantiano, esto es, como ese horizonte infinito -aunque implicado o determinado intencionalmente- de experiencias posibles. 
nariamente o en persona como lo dado en sí mismo de lo dado meramente de un modo vacío.

En el primer sentido expuesto, la claridad de la anticipación reclama, entonces, su propia evidencia en cuanto se presenta con la necesidad de la implicación intencional, es decir, como verdad posible. En la necesidad de la posibilidad como anticipación infinita pero determinada intencionalmente está la evidencia clara anticipativa. Aquí se manifiesta lo que ya se había expuesto en Ideas $I^{54}$ : a partir de una comprensión estática que tiene su origen en las Investigaciones lógicas, la expresión se constituye en el único medio de acceso al significado, de tal manera que el significado comprendido logísiticamente se convierte en el marco de la verdad posible. La diferencia en la Lógica con respecto a Ideas radica en que ahora es consciente y explícito este paso metodológico, pues se procede según "la intención viva de los lógicos" ${ }^{55}$, más precisamente, según el objetivo de las ciencias formales:

Hasta aquí hemos seguido el método de exponer sistemáticamente la estructura teleológica de la idea de la lógica; gracias a él hemos desarrollado y depurado, en alguna medida, por lo menos una de esas estructuras: la idea de la analítica formal referida exclusivamente a los juicios (en cuanto significaciones puras). [...] Siguiendo la estructura de las significaciones ideales, pudimos dividir en tres estratos el sentido "innato" -por así decir- de la lógica tradicional y exponer luego las tres disciplinas, que se fundan una sobre otra en la analítica pura de los juicios. ${ }^{56}$

Y ante la pregunta por la verdad posible -en el marco de una lógica de la verdad-, que implica la pregunta por la adecuación a objetos, se advierte una diferenciación fundamental:

\begin{abstract}
El predicado "verdad" se refiere, sin duda, a juicios y solamente a juicios, así adoptemos el concepto lato o el concepto estricto de juicio (apófansis) arriba señalado. Pero mientras nos atengamos a la mera evidencia distinta y a lo que permite identificar en ella el rubro de "juicio", queda excluida de seguro cualquier contradicción (cualquier contrasentido analítico); aún es posible, empero, un contrasentido referente a las cosas y cualquier otra no-verdad. En efecto, entonces hacemos abstracción de toda operación de clarificación, de remisión a la posibilidad y verdad de las cosas; con otras palabras: de toda cuestión acerca de la verificación. ${ }^{57}$
\end{abstract}

${ }^{54}$ Explícitamente en una nota al pie del $\S 124$ de Ideas I: "De hecho fue éste [los problemas de la expresión y la significación] el camino por el que se esforzaron por entrar las Investigaciones lógicas en la fenomenología. Un segundo camino que partía del lado opuesto, a saber, del lado de la experiencia y de los datos sensibles, y que el autor siguió igualmente desde comienzo del último decenio del siglo pasado, no encontró su plena expresión en dicha obra".

${ }^{55}$ Hua XVII, 14.

${ }^{56}$ Ibidem, p. 79.

57 Ibidem, p. 70. 
El resaltado, que pertenece al mismo Husserl, hace patente el contraste entre un contrasentido analítico y un posible contrasentido fundado en las cosas. La lógica de la verdad permanece dentro de un marco meramente formal en estos análisis. Y, mientras tal sea el caso, se limitará y se regirá por los principios de la lógica de la consecuencia, es decir, "no puede alcanzar otros conocimientos sobre la verdad posible que los inmediatamente ligados a la analítica pura de la no contradicción" ${ }^{58}$; de allí que la lógica de la verdad no aporte ningún incremento en disciplinas que deban ser agregadas a la lógica formal, sino que trae consigo sólo el interés de conocimiento ${ }^{59}$, y esto quiere decir que permanece dentro del tema propio de la lógica formal: el sentido. Lo que aquí debe comprenderse es que, si bien todo juzgar está referido a objetos, no sólo un objeto-sustrato, una cualidad o situación objetiva pueden ser objetos, sino también un sustrato mentado en cuanto mentado, una cualidad mentada en cuanto mentada, pues "las entidades mentadas [Vermeintheiten] en cuanto tales son objetos de una región peculiar, forman un campo de objetos cerrado en sí $^{\prime 60}$. La lógica, en principio, no se interesa por la verdad real. Quiso ser desde un comienzo teoría de la ciencia y reflexionar acerca de la intencionalidad científica, para lo cual constituyó la esfera judicativa en su propio campo temático, a fin de poder ejercer la crítica que creara la ciencia auténtica. Es por eso que asumía los conocimientos de las ciencias, sus juicios, como meras pretensiones de saber, es decir, como meras menciones sobre las que habría de aplicarse la crítica para luego poder atribuir con justificación a sus juicios el predicado de verdaderos.

Ahora bien, una lógica verdaderamente filosófica ${ }^{61}$, esto es, que pretende convertirse en teoría de la ciencia, tiene que tener al menos la intención de pensar su posibilidad de aplicación y de determinación de objetividades concretas para poder convertirse en una ontología formal. El verdadero sentido de una

58 Ibidem, p. 77.

${ }^{59}$ Cfr. Dieter Lohmar, op. cit., p. 59 y nota 84.

${ }^{60}$ Hua XVII, 136.

61 "Lógica filosófica" y "Lógica verdaderamente filosófica" son expresiones que utiliza Husserl [cfr. especialmente la Introducción] para referirse a la Lógica que asume una perspectiva fenomenológicotrascendental, es decir, aquella que hace suya "la idea de que todos los problemas de sentido dirigidos subjetivamente, que la ciencia y la lógica se plantean y deben plantearse, no son problemas de la subjetividad humana natural, esto es, problemas psicológicos, sino problemas de la subjetividad trascendental, en el sentido (por mí introducido) de la fenomenología trascendental", y, así, pueda convertirse en "una teoría de la ciencia que exponga en todas sus facetas la posibilidad esencial de la ciencia auténtica y pueda así servir de guía al desarrollo de esa ciencia". Ibidem, p. 17. 
analítica formal que quiere ser teoría de la ciencia, "es ser una ciencia de las formas categoriales posibles, en las que puedan existir verdaderamente las objetividades-sustratos" ${ }^{62}$. Y éstas son objetividades formadas categorialmente -conceptos ontológicos, no apofánticos-, lo cual equivale a un juicio cumplido, de tal modo que aquí tiene lugar una síntesis de coincidencia por medio de la cual el que juzga, estando dirigido a objetos, alcanza la posesión evidente de las cosas mismas; dicho de otro modo, resulta dado lo mismo que se menciona. Aquí la evidencia realiza y aprehende las objetividades-sustrato ${ }^{63}$. Las realiza, sí, como unidad sintética de otras evidencias fundantes. Ya se había reconocido fundamentalmente en las Investigaciones lógicas ${ }^{64}$ que las objetividades categoriales son objetos de grado superior y que sólo pueden constituirse en una complexión de actos fundados unos sobre otros en una gradación de evidencias. De ello resulta un doble sentido de evidencia: por un lado, la "situación objetiva verdaderamente existente" y, por otro, el "darse la corrección de la mención judicativa" ${ }^{\prime 65}$. En el primer caso, la evidencia se refiere a los objetos dados categorialmente de modo pleno, mientras que, en el segundo, la evidencia se refiere a los sentidos del juicio en vistas a su conformidad con la objetividad categorial dada, es decir, en vistas a la primera evidencia. Son la claridad de la posesión y la claridad de la anticipación.

\section{Segunda Sección: LA PERSPECTIVA Genética}

Toda formación lógica, objetiva en el sentido que se viene considerando, tiene su correlato subjetivo en las intencionalidades constituyentes. No debe olvidarse esa doble dirección de la lógica, ya anticipada en el § 8: "Se trata siempre de operaciones de la razón en un doble sentido: por un lado, las actividades y habitualidades operantes, por el otro, los resultados permanentes logrados por ellas" ${ }^{\prime 66}$. La Sección Segunda de la Lógica se centra en la temática de dirección subjetiva y tiene como fin investigar las operaciones de la subjetividad que entran en juego en la constitución de objetividades.

\footnotetext{
${ }^{62}$ Ibidem, p. 151.

${ }^{63}$ Cfr. Idem. "[D]ie Evidenz verwirklicht und erfaßt sie selbst".

${ }^{64}$ Especialmente en la Sección Segunda de la Sexta Investigación lógica.

${ }^{65}$ Hua, 153.

${ }^{66}$ Ibidem, pp. 36s.
} 
Esta perspectiva de análisis mostrará un punto fundamental: que la evidencia es análoga en toda objetividad, ya sea real, irreal o ideal, de la experiencia interna o externa. Estos rasgos esenciales pertenecen a la evidencia de toda objetividad en cuanto objetividad. A esta conclusión arriba Husserl luego de renovar su crítica contra el psicologismo, que amenaza siempre la distinción entre el juzgar y lo juzgado, y de mostrar la identidad de los objetos ideales [§§ 55-57] ${ }^{67}-\mathrm{u}$ objetividades del entendimiento [Verstandesgegenständlichkeiten], como las denomina en Experiencia y juicio ${ }^{68}$, , pues es la identidad [Identität] e identificabilidad [Identifizierbarkeit] el carácter objetivo de toda objetividad y, en consecuencia, lo que se "experimenta", lo que se "ve"69 y que resulta en una posesión evidente. Así se ve ampliado también el concepto de experiencia, ya que la posibilidad de identificación es el correlato esencial de todo objeto de experiencia. En consecuencia, "el sentido de 'experiencia' se determina como la aprehensión y posesión evidentes de un dato individual (inmanente o real) 'él mismo'"'70.

La analogía de la evidencia de todas las objetividades radica en la posibilidad de ser identificadas en repetidas y diversas experiencias según sus propios modos de aprehensión y posesión del objeto mismo. La diferencia en el caso de los objetos irreales está dada en la ausencia esencial de una temporalidad que los individualice de manera original ${ }^{71}$, pues la forma temporal de las objetividades o elementos de nivel inferior no cumplen en ellos una función de representación, es decir que la temporalidad que asumen los objetos de grado superior (objetividades irreales) no es esencial en cuanto no determina su individuación ${ }^{72}$.

La mencionada analogía de la evidencia de toda objetividad tiene su fundamento en una intencionalidad continuamente actuante que permite una referencia "cruzada" en diversas direcciones, ya sea en sentido temporal, ya en cuanto a su modalización, ya con respecto a las diversas formas de presentifi-

67 "[E]s una evidencia original [ursprüngliche Evidenz] que los juicios, deducciones, etcétera, formados en actos reiterados, iguales o semejantes entre sí, no son meramente iguales o semejantes, sino que son los mismos juicios, raciocinios, etcétera, numéricamente idénticos." Ibidem, § 57 b, p. 162.

${ }^{68}$ Cfr. Edmund Husserl, Experiencia y juicio, México, U.N.A.M., 1980, §§ 58-65. La traducción española es de Jas Reuter.

${ }^{69} \mathrm{Cfr}$. Hua XVII, 163.

${ }^{70}$ Ibidem, p. 164.

${ }^{71}$ Aunque puedan aceptar, sin embargo, una tal referencia. Cfr. Ibidem, p. 164, nota al pie.

$72 \mathrm{Cfr}$. Edmund Husserl. Experiencia y juicio, p. 284. La paginación del texto alemán corresponde a Erfahrung und Urteil, Hamburg, Felix Meiner Verlag, 19997, p. 310. 
cación [Vergegenwärtigung] (pura fantasía, ilusión, recuerdo, etc.) o de conciencia reproductora, en referencia siempre a la presentación [Gegenwärtigung] propia de la percepción. Justamente porque la sola percepción interna no es una operación plenamente objetivante ${ }^{73}$, esta referencialidad cruzada propia de una vida intencional y no de un acto aislado permite comprender la evidencia como "la operación intencional de darse las cosas mismas" ${ }^{\prime 74}$; operación intencional en cuanto que la posibilidad de la identidad $-\mathrm{y}$, consecuentemente, de la evidencia- implica que toda percepción de alguna manera mienta juntamente la posibilidad de ser repetida, de volver sobre ella indefinidamente, esto es, de ser reconocida. Lohmar explica que "[I]a evidencia remite ya en este sentido a una 'vida' en efectuaciones intencionales del sujeto, es decir, a una historia de experiencia pasada sedimentada, a mis expectativas (en correspondencia con mis experiencias de la misma especie) y también a mi conciencia de otras posibilidades de obrar, de percibir y de experimentar, eventualmente incluso a la comunicación con otros"75.

Hay evidencia cuando hay una mismidad dada, cuando la objetividad es "vista" ella misma. Por eso la evidencia es la conciencia primordial ${ }^{76}$, la conciencia en que es dada una identidad, pues en ella se aprehende la cosa misma de modo original en contraste con los modos de aprehensión simbólicos (imagen), signitivos o menciones vacías en general. Pero, a su vez, toda evidencia, como conciencia primordial, requiere -podríamos decir- de un darse juntamente posibilidades en diversos sentidos ${ }^{77}$ para poder constituirse ella misma.

La noción de "función" es otro recurso descriptivo que permite comprender la configuración propia de toda evidencia y que nosotros denominamos "modal" atendiendo a la necesidad esencial con que se presenta un entramado de posibilidades como contexto intencional en el que puede constituirse la evidencia en cuanto unidad sintética: "las evidencias son funciones que fungen en sus contextos intencionales"78.

\footnotetext{
${ }^{73} \mathrm{Cfr}$. Hua XVII, 165 y 291. Husserl sostiene que sería un error considerar la presencia de un dato o "percepto inmanente" en una vivencia constituyente como plenamente constituido en cuanto objeto. Éste es sólo el primer nivel de un objeto y no un objeto en sentido propio.

${ }^{74}$ Ibidem, p. 166.

75 Dieter Lohmar, op. cit., pp. 120 s.

${ }^{76}$ Cfr. Hua XVII, 166.

77 Lo que denominamos más arriba como una especie de referencialidad "cruzada" y que manifiesta la constitución modal de la experiencia en el sentido que planteamos.

${ }^{78}$ Hua XVII, 291.
} 
Ya mencionamos antes que el acto de darse las cosas mismas, como cualquier vivencia intencional singular, es una función en el contexto universal de la conciencia. Su operación no está pues conclusa en su singularidad; tampoco lo está como acto de darse las cosas mismas, como evidencia, por cuanto su intencionalidad propia puede implícitamente "exigir" ulteriores actos de darse las cosas mismas, puede remitir a ellos para consumar su operación objetivante. ${ }^{79}$

El darse mismo de las cosas mismas no es una operación singular sino un contexto o configuración intencional en el que hay implícitas infinitas aunque determinadas relaciones e implicaciones que permiten lo que puede denominarse "evidencia en cuanto vivencia singular" 80 . "Así, la evidencia es un modo universal de la intencionalidad referido a la vida de conciencia en su conjunto" ${ }^{\prime 1}$.

A estas determinaciones generales del contexto intencional propio de toda evidencia en cuanto el darse un objeto él mismo -o, noéticamente, como poseer el objeto mismo ${ }^{82}$ - corresponden rasgos específicos, a su vez propios de la estructura intencional del darse de cada especie de objetividad -según la región y categoría-, como ya se trató en las Investigaciones lógicas y en Ideas. "La categoría de objetividad y la categoría de evidencia son correlatos"83, de donde surge la determinación de la evidencia según un estilo [Stil] y una especie o clase [Art]: "A toda especie fundamental de objetividades [...] corresponde una especie fundamental de 'experiencia', de evidencia, como también una especie fundamental de estilo de evidencia" ${ }^{84}$. Un objeto de la naturaleza, en cuanto espacial, es dado él mismo según un estilo propio, diverso al de una objetividad irreal o a un signo. A su vez, lo percibido, en cuanto percibido, tiene su propio "estilo" de evidencia distinto al "estilo" de la rememoración. Aquí deberían hacerse las siguientes distinciones: un objeto espacial, por ejemplo, prescribe su manera propia de ser dado él mismo en la percepción. Si lo consideramos desde el punto de vista del nóema completo, diremos que el sentido noemático presenta una evidencia según un estilo exigido por el objeto, por un lado,

79 Ibidem, p. 168.

${ }^{80}$ Ibidem, p. 169.

${ }^{81}$ Ibidem, p. 168.

${ }^{82}$ Cfr. Ibidem, p. 169.

${ }^{83}$ Idem.

${ }^{84}$ Idem. "Zu jeder Grundart von Gegenständlichkeiten [...] gehört eine Grundart der 'Erfahrung', der Evidenz und ebenso des intencional indizierten Evidenzstiles...". Corregimos un detalle de la traducción española de Villoro que consideramos se corresponde más precisamente con el texto alemán. Traducción de Villoro: "A toda especie fundamental de objetividades [...] corresponde una especie fundamental de 'experiencia' evidente; corresponde asimismo una especie fundamental de estilo de evidencia...". 
1) en el cómo de sus determinaciones (de acuerdo con la región o categoría a la que pertenece), y, por otro lado,

2) en el cómo de sus maneras subjetivas de darse (en la percepción o en presentificaciones).

Ahora bien, si el estilo de la evidencia se corresponde con la categoría del objeto (1), hay, a su vez, rasgos propios de la evidencia que se ven determinados a partir de la manera subjetiva de darse esa objetividad (2), es decir que, en realidad, hay determinaciones de la evidencia que no tienen su fundamento de modo inmediato en la categoría objetiva sino en la manera subjetiva de darse. Si bien Husserl no se refiere a este rasgo de la evidencia con una denominación específica y, de hecho, suele considerarlo también como "estilo", se trata de una determinación diversa. Tomemos un ejemplo para advertirlo más claramente: si contemplo un paisaje, su ser espacial y sensible perteneciente a la experiencia externa determina su dación de sí mismo originaria en la percepción según una sucesión de escorzos, cada uno de los cuales es dado como una síntesis de apercepciones que incluyen momentos plenos junto con un horizonte de anticipaciones vacías que codeterminan el ingreso y transcurso inmediato y mediato de posibles cumplimientos coherentes. Este mismo paisaje percibido, en cuanto recordado, ofrece una nueva manera de darse precisamente como rememoración. En cuanto objetividad sensible recordada decimos que presenta un carácter reproductivo con relación o referencia esencial al presente actual. Un signo, por ejemplo, posee un estilo de dación en sí mismo en la percepción diverso al de un objeto espacial: lo dado en la percepción de modo pleno (incluso con su espacialidad) es, en realidad, mención de otra cosa. Sin embargo, el signo posee la misma determinación que un paisaje en su manera de darse en cuanto recordado. Por otro lado, un objeto ideal, por ejemplo un número, no es dado según el mismo estilo de evidencia que un objeto de la percepción externa. Un número es aprehendido en una operación análoga a la experiencia, de tal manera que es identificado, aunque, por su propia esencia, no es individualizado según una temporalidad que le sea constitutiva -como ya se ha dicho. En este sentido, una objetividad ideal podrá ser recordada al modo de un objeto sensible si se hace de ella una individualidad ${ }^{85}$, si bien esta temporalidad no le

\footnotetext{
${ }^{85}$ Es decir, si se atiende a la vivencia en que es dada, al juzgar, al colegir, al contar, y no al juicio, al número o a las verdades en ellos constituidas.
} 
será esencial para su constitución en cuanto identidad ideal. De allí que toda "rememoración" de una objetividad ideal sea más propiamente una percepción, en cuanto cada vez es dada la misma objetividad, numéricamente idéntica, y no una reproducción de una objetividad dada previamente de modo originario en un presente ya transcurrido.

Resulta, por ello, impreciso considerar -como lo hace Lohmar- la determinación del estilo de la evidencia a partir de las diversas maneras [Weisen ${ }^{86}$ ] de conciencia, "otro aspecto importante de la evidencia es el estilo de la obtención de evidencia (sic), [...]. Así, una rememoración, por ej., encuentra su confirmación en el surgir involuntario de imágenes del recuerdo y de la reconstrucción de las conexiones entre los sucesos recordados, etc. Con ello tiene un estilo de evidencia distinto a la percepción, que, por ej., se adelanta a una posible repetición de la dación [Gegebenheit] sensible ${ }^{\prime 87}$, pues las diferencias en la evidencia de las diversas maneras de conciencia son diferencias propias de cada manera en cuanto tal, que, si bien se desprenden esencialmente del estilo de evidencia que determina cada objeto, poseen, por su parte, rasgos comunes en cuanto manera subjetiva de darse:

La multiplicidad de percepciones, de recuerdos posibles de otras vivencias intencionales en general, que se refieren y pueden referirse de modo "concordante" a una y la misma cosa, tienen, con toda su inmensa complicación, un estilo esencial enteramente particular, idéntico para cada cosa, que sólo se particulariza de una cosa individual a otra. Asimismo las diversas maneras de conciencia que pueden hacer presente a la conciencia alguna objetividad ideal y que deben reunirse en la unidad de una conciencia sintética de esa objetividad, tienen un estilo determinado, conforme con la esencia de esa especie de objetividad. ${ }^{88}$

\footnotetext{
${ }^{86}$ Villoro traduce Weise por 'modo'. Dada la importancia que reviste el concepto de modo en nuestra exposición, preferimos utilizar el término 'manera' en los contextos y citas en que aparece Weise y reservar el término 'modo' para Modus.

87 Dieter Lohmar, op. cit., p. 120.

${ }^{88}$ Hua XVII, 253. El resaltado es del mismo Husserl. En esta cita, como anticipamos, se denomina estilo a la determinación de la evidencia correspondiente a la manera subjetiva de darse la objetividad. Decimos, entonces, que es "impreciso" considerar el estilo de la evidencia como determinado de manera inmediata a partir de las maneras de conciencia, porque se trata de una determinación mediata que tiene su origen en la categoría del objeto: de allí que la "rememoración" de un objeto ideal no puede serlo propiamente, sino que consiste en una percepción -como se explicó más arriba. En Ideas I se expuso precisamente esta determinación de la evidencia que también se recupera aquí. En la Lógica se enriquece con más descripciones. Diríamos que el estilo de la evidencia implica una consideración objetiva y una subjetiva, pero siempre desde el sentido fundamental de esta determinación de la evidencia que parte de la categoría o especie de objetividad. La consideración objetiva del estilo de la evidencia revela la dación del momento noemático del objeto en el cómo de sus determinaciones, y la consideración subjetiva, la dación del objeto en el cómo de sus maneras subjetivas de darse, que viene siempre "mediada" por la categoría objetiva. El problema radica en que Husserl no utiliza un término específico para esta consideración "subjetiva" de la determinación de la evidencia, y le llama alguna vez "estilo", cuando, en realidad, ya había denominado "estilo" a la determinación de la evidencia que tiene su origen en la categoría de la objetividad. De allí que, decir-como Lohmar- que el estilo de la evidencia se ve
} 
En consecuencia, si cada objeto determina el estilo de su dación, lo determina también en cuanto original, pues "[I]a evidencia, entendida con plena generalidad, no es justamente más que la manera de conciencia que, construyéndose en una serie de niveles extraordinariamente complejos, ofrece su objetividad intencional a modo de lo originalmente dado" ${ }^{89}$, de tal manera que cualquier modificación intencional remite esencialmente, en cuanto modificación, a lo no modificado ${ }^{90}$, a la vez que toda conciencia original puede constituirse como tal porque es dada juntamente la posibilidad de su modificación, en primer lugar como rememoración (identificabilidad de lo mismo en una conciencia reproductiva) o presentificación, pero, también, como cancelación o también como modalización. Noéticamente diremos que cada una de estas maneras de conciencia ofrece su objetividad con una evidencia que le es propia, mientras que, noemáticamente diremos que es "[u]no y el mismo objeto [el que] puede a priori estar ante la conciencia de muy diversos modos (según ciertos tipos esenciales: percepción, rememoración, conciencia vacía, etc. $)^{\prime 91}$, obrando precisamente esta unidad del objeto mentado -desde una perspectiva estáticacomo guía u orientación del proceso de dación en sí mismo ${ }^{92}$. Pero la remisión esencial de toda conciencia a otras maneras de conciencia para la constitución de su propia evidencia permite o, más bien, reclama un análisis del fundamento de esta remisión: la evidencia de cada experiencia muestra una génesis temporal constituyente. Cada vivencia tiene su historia en "la unidad inmanente de la temporalidad de la vida"93. Y "génesis" remite siempre a originalidad del darse, que, conformándose según la región o categoría objetiva, se presentará genéticamente según un tipo [Typus] o típica [Typik] de experiencia. Esto quiere decir que toda experiencia es o bien repetición o bien presentación de algo semejante a lo ya experimentado.

Pero en cualquier caso toda conciencia ocurre, por esencia, en una particular multiplicidad de conciencia [Bewußtseinsmannigfaltigkeit] inherente a ella, en una infinitud sintética abierta de maneras posibles de tener conciencia de lo mismo: multiplicidad que, por así decirlo, tiene su centro teleológico en la "experiencia" posible. Ésta designa primero un horizonte de evidencia que cumple las menciones con la cosa misma

determinado a partir de las maneras subjetivas de darse el objeto, sería incorrecto o, al menos, "impreciso".

\footnotetext{
89 Hua XVII, 176.

${ }^{90}$ Cfr. Ibidem, p. 315.

${ }^{91}$ Idem.

92 Cfr. Ibidem, p. 316.

${ }^{93}$ Idem.
} 
anticipada como algo que "ha de realizarse". Pero por esencia queda abierta la posibilidad contraria, la del engaño: la cancelación de lo anticipado al darse "otra cosa en lugar de ésa"; lo que indica una forma contraria de la multiplicidad centrada. ${ }^{94}$

Es este contexto u horizonte de la experiencia posible el que es dado juntamente con toda experiencia en sentido estricto $y$, en un sentido amplio, también "experimentado" y dado, en consecuencia, con "evidencia" o, mejor dicho quizás, conformando la misma evidencia de lo dado. Podemos advertir, entonces, que toda evidencia, en cuanto tal, esto es, en cuanto darse algo en sí mismo o lo dado en sí mismo (noética o noemáticamente considerado, respectivamente), consiste en una configuración intencional de conciencia tal que funcionalmente $-i$. e., como función- y, por ello, como una relación determinada entre variables, se constituye como un contexto de dación múltiple que podríamos caracterizar como de necesidad abierta o, en otros términos, como de posibilidad determinada; configuración, entonces, que puede entenderse como modal en cuanto que se constituye esencialmente de manera referencial según operaciones de síntesis y no como un campo de sentido extensionalmente uniforme, cerrado y absoluto.

La necesidad abierta o posibilidad determinada que caracteriza a toda conciencia que da con evidencia se fundamenta en su configuración típica o tipificante. Si bien Husserl recurre al tipo como a un elemento de descripción primariamente empírico, creemos que vale como "esquema" descriptivo para toda conciencia. Ya mencionamos cómo el autor de la Lógica se refiere a la "experiencia" de objetos ideales sosteniendo que incluso se "ven" y que su evidencia es, pues, análoga a la de los objetos reales ${ }^{95}$. Lo tipificante en el caso de la experiencia de objetos ideales no radica en el enriquecerse o variar el tipo concreto operante, sino en el predelineamiento de tipos constituidos en génesis ante-

${ }^{94}$ Idem: "Jedenfalls aber steht jedes Bewußtsein wesensmäßig in einer besonderen, ihm zugehörigen Bewußtseinsmannigfaltigkeit, in einer synthetischen offenen Unendlichkeit möglicher Bewußtseinsweisen von Demselben - einer Mannigfaltigkeit, die aber sozusagen ihre teleologische Mitte hat in der möglichen 'Erfahrung'. Das bezeichnet zunächst einen Horizont erfüllender Evidenz mit dem als 'zu verwirklichend' antizipierten Es-selbst. Dabei bleibt aber wesensmäßig offen die Gegenmöglichkeit enttäuschender Aufhebung des Antizipierten im 'Statt-dessen-Anderes', was eine Gegengestalt der zentrierten Mannigfaltigkeit anzeigt." Corregimos la traducción de Villoro que presenta varios errores. Versión de Villoro: "Pero en cualquier caso toda conciencia ocurre, por esencia, en una particular multiplicidad de actos de conciencia inherentes a ella, en una infinitud sintética abierta de modos posibles de tener conciencia de lo mismo: multiplicidad que, por así decirlo, tiene su centro teleológico en la "experiencia' posible. Ésta designa primero un horizonte de evidencia que cumple las menciones con la cosa misma anticipada como algo que 'ha de realizarse'. Pero por esencia queda abierta la posibilidad contraria, la del engaño: la cancelación de lo anticipado al darse 'otra cosa en lugar de ésa'; lo que indica una forma contraria de multiplicidad centrada en la experiencia".

${ }^{95} \mathrm{Cfr}$. in supra: "Esta perspectiva de análisis mostrará...". 
riores que fundan la experiencia actual ${ }^{96}$.

En el desarrollo de la noción del tipo y de la apercepción tipificante que aparece fundamentalmente en el $\S 8$ y también en el $\S 26$ de Experiencia y juicio queda claro que la evidencia, en cuanto dación de lo mentado en sí mismo, presupone una intención anticipativa como forma teleológica para la posibilidad de todo cumplimiento. Ahora bien, la anticipación o expectativa, por ser el marco de posibilidades predelineadas, delata, a su vez, una motivación o ser condicionado del que emergen dichas posibilidades precisamente como motivadas $y$ no como aleatorias o indefinidas. La receptividad de la conciencia y su constitución resultan, pues, de una génesis de conservación de experiencia. Nunca se comienza de cero en el camino de la experiencia. Lo desconocido es dado siempre dentro de los límites tipificantes más generales de lo conocido, y ello porque sólo así es esencialmente posible lo desconocido mismo, que motivará el cumplimiento de la mención vacía o la modificación y consecuente surgimiento de nuevos tipos como especificaciones y transformaciones de los que ya estaban en juego.

La apercepción tipificante y el tipo, entendido como su objetivación, se manifiestan, en consecuencia, como la explicación genética -en el sentido fuerte de síntesis explicativas, como se comprende en Experiencia y juicio- de toda evidencia.

${ }^{96} \mathrm{Cfr}$. Hua XVII, 317: Pero nuestra representación de cosas, incluso nuestra visión de cosas en una sola mirada [...], remite en el análisis genético intencional al hecho de que, en una génesis anterior primordialmente fundante, se haya realizado el tipo "experiencia de la cosa" y por ende se haya fundado ya, por primera vez, la categoría de "cosa para nosotros". Pero esto sucede por esencia -como es patente- con cualquier categoría objetiva entendida en su sentido más amplio, sucede con la de dato "inmanente" de sensación, pero también con cualquier objetividad del nivel de las objetividades mentales, de las formaciones judicativas, con cualquier objetividad de las teorías verdaderamente existentes, incluso con las formaciones axiológicas y prácticas, los proyectos prácticos, etc. 